\title{
Role of Entropy in Domain Wall Motion in Thermal Gradients
}

\author{
F. Schlickeiser, U. Ritzmann, D. Hinzke, and U. Nowak \\ Fachbereich Physik, Universität Konstanz, D 78457 Konstanz, Germany
}

\begin{abstract}
Thermally driven domain wall (DW) motion caused solely by magnonic spin currents was forecast theoretically and has been measured recently in a magnetic insulator using magneto optical Kerr effect microscopy. We present an analytical calculation of the DW velocity as well as the Walker breakdown within the framework of the Landau Lifshitz Bloch equation of motion. The temperature gradient leads to a torque term acting on the magnetization where the DW is mainly driven by the temperature dependence of the exchange stiffness, or in a more general picture by the maximization of entropy. The existence of this entropic torque term does not rest on the angular momentum transfer from the magnonic spin current. Hence, even DWs in antiferromagnets or compensated ferrimagnets should move accordingly. We further argue that the entropic torque exceeds that of the magnonic spin current.
\end{abstract}

Spin caloritronics is a new field of research focused on the combined transport of spin, charge, entropy, and energy in magnetic systems [1]. A good example for a spin caloritronic phenomenon is the spin Seebeck effect, the occurrence of spin currents or spin accumulation due to temperature gradients, which was discovered first in metallic ferromagnets [2]. Later on, this effect was also found in dilute magnetic semiconductors [3] and even in insulators [4]. In the latter case the existence of the spin Seebeck effect can only be explained by pure magnonic spin currents not resting on any charge transport.

Spin caloritronic phenomena call for an exploitation in magnetic devices. In Ref. [5] the existence of thermally driven domain wall (DW) motion in a temperature gradient was demonstrated by computer simulations based either on the stochastic Landau-Lifshitz-Gilbert equation of motion for an atomistic spin model or-in a more micromagnetic picture - on the Landau-Lifshitz-Bloch (LLB) equation of motion for a thermally averaged spin polarization. The microscopic explanation for DW motion in temperature gradients rests on the diffusive motion of magnons from the hot end of a nanowire towards the colder end [6] and the transfer of angular momentum pushing the wall in the direction opposite to the magnonic spin current. Theoretical investigations based on these arguments give estimates for the DW velocity which can be achieved [7-10].

In this work we will focus on a more thermodynamic point of view. At finite temperature $T$ a DW is a thermodynamic object where the free energy $\Delta F(T)$ is the thermodynamic potential that is minimized. The free energy of the DW can be expressed as the difference between a magnetic system with DW minus the free energy of the same system without DW [11]. It is connected to the internal energy $\Delta U$ of the wall and its entropy $\Delta S$ via $\Delta F=\Delta U-T \Delta S$. The temperature dependence of the different thermodynamic potentials are illustrated in
Fig. 1 for a DW in a high anisotropic material. The calculations are from Ref. [11] for an atomistic spin model for FePt. However, the general features of a DW free energy do not depend on the material under investigation. While the internal energy $\Delta U$ increases, the free energy of the DW is a monotonically decaying function of temperature. This is due to the fact that the entropy of the DW increases with temperature due to the increasing spin disorder following the thermal excitation. Note that the maximum of $\Delta S$ and $\Delta U$ is slightly below the Curie temperature $T_{C}$, where the DW becomes linear [11-13]. The increase of the entropy $\Delta S(T)$ of the wall and the decrease of the free energy $\Delta F(T)$ with temperature alone must lead to DW motion in a temperature gradient, as long as the temperature gradient is sufficiently small so that equilibrium thermodynamics can be applied. To minimize its free energy, the DW must move towards regions with higher temperatures. In the following we will calculate DW motion analytically based on these thermodynamic arguments within the framework

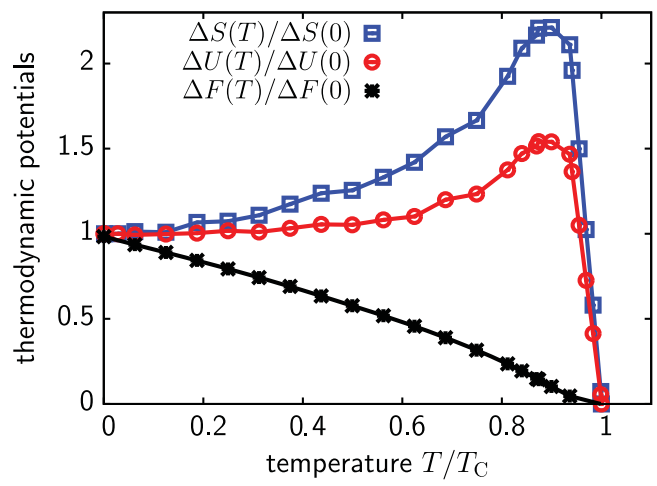

FIG. 1 (color online). Temperature dependence of different thermodynamic potentials of a DW in a high anisotropic material [11]. The increase of the entropy $\Delta S$ leads to a monotonically decaying free energy $\Delta F(T)$ as a function of temperature. 
of the LLB equation. We show that the strong decay of the exchange stiffness plays the main role in minimizing the free energy and thereby pushing the DW into the hotter region. In order to verify our analytical approach for the velocity of the DW as well as the Walker threshold we also present numerical simulations. Interestingly, the above argument does not rest on the existence of magnonic spin currents with angular momentum transfer so that it should apply more generally, e.g., to antiferromagnets or ferrimagnets at the compensation point as well. Finally, we compare our results with those resting on magnonic spin transfer torque showing that the entropy driven DW motion exceeds the magnon driven one [7-10].

A framework for thermal micromagnetic calculations was recently developed [14] and is meanwhile well established in the context of ultrafast spin dynamics $[15,16]$, spin torque [17,18], vortex core reversal [19], and spin caloritronics [5]. It rests on the LLB equation of motion which was derived earlier by Garanin [20] to describe the dynamics of a thermally averaged reduced magnetization vector $\mathbf{m}_{i}$ via the Fokker-Planck equation. The LLB equation does not conserve the length of the magnetization vector, it rather allows for longitudinal fluctuations of the magnetization in space and time and all relevant magnetic material parameters such as exchange stiffness, anisotropy, and equilibrium magnetization become functions of temperature that are well defined in terms of their microscopic degrees of freedom. The LLB equation reads

$$
\begin{aligned}
\dot{\mathbf{m}}_{i}= & -\gamma \mathbf{m}_{i} \times \mathbf{H}_{\mathrm{eff}}^{i}+\frac{\gamma \alpha_{\|}}{m_{i}^{2}}\left(\mathbf{m}_{i} \cdot \mathbf{H}_{\mathrm{eff}}^{i}\right) \mathbf{m}_{i} \\
& -\frac{\gamma \alpha_{\perp}}{m_{i}^{2}} \mathbf{m}_{i} \times\left(\mathbf{m}_{i} \times \mathbf{H}_{\mathrm{eff}}^{i}\right)
\end{aligned}
$$

Besides the usual precession and relaxation terms of the Landau-Lifshitz-Gilbert equation, the LLB equation contains another term which controls longitudinal relaxation. For $T \leq T_{C}$ the temperature dependent longitudinal and transverse damping parameters $\alpha_{\|}$and $\alpha_{\perp}$ are connected to the atomistic damping parameter $\lambda$ via $\alpha_{\|}=\lambda 2 T /\left(3 T_{C}\right)$ and $\alpha_{\perp}=\lambda\left[1-T /\left(3 T_{C}\right)\right]$. The effective fields $\mathbf{H}_{\text {eff }}$ are given by $[14]$

$$
\begin{aligned}
\mathbf{H}_{\mathrm{eff}}^{i}= & -\frac{k m_{x}^{i} \mathbf{e}_{x}+m_{y}^{i} \mathbf{e}_{y}}{\tilde{\chi}_{\perp}}-\frac{2 A}{m_{e}^{2} M_{s} \Delta^{2}} \sum_{j}\left(\mathbf{m}_{j}-\mathbf{m}_{i}\right) \\
& +\frac{1}{2 \tilde{\chi}_{\|}}\left(1-\frac{m_{i}^{2}}{m_{e}^{2}}\right) \mathbf{m}_{i}+\mathbf{H}
\end{aligned}
$$

Here, the first term represents the anisotropy field which makes the $z$ axis the easy axis of the model and $k<1$ is a factor which breaks the $x-y$ symmetry so that $x$ is the intermediate and $y$ the hard axis of the model. The second term is the exchange field where $M_{s}$ is the zero-temperature saturation magnetization and $\Delta$ is the cell size of the mesh.
Within the framework of the LLB equation the temperature dependence of the effective fields is defined via thermodynamic equilibrium functions, namely, the reduced equilibrium magnetization $m_{e}(T)$, the exchange stiffness $A(T)$, and the susceptibilities $\tilde{\chi}_{\|}(T)$ and $\tilde{\chi}_{\perp}(T)$, which have been calculated in [14] for FePt based on atomistic spin model simulations. For the current work we rescaled these functions to match the material properties of a CoPd multilayer system [21,22]. These functions are shown in Fig. 2. The corresponding zero-temperature material parameters are a saturation magnetization of $M_{s}=8.2 \times 10^{5} \mathrm{~A} / \mathrm{m}, \quad$ an anisotropy constant $K_{0}=$ $6 \times 10^{5} \mathrm{~J} / \mathrm{m}^{3}$, and an exchange stiffness $A(0)=$ $2.3 \times 10^{11} \mathrm{~J} / \mathrm{m}$, where we have used the relation $\tilde{\chi}_{\perp}(0)=M_{s}^{2} \mu_{0} /\left(2 K_{0}\right)$. Even though details [e.g., the exponents $\kappa$ and $\epsilon$ describing $A(T) \propto m_{e}^{\kappa}$ and $K(T) \propto m_{e}^{\epsilon}$ ] do depend on structural and material details [23], the functional forms are rather general for any ferromagnetic system. In the following we model a nanowire. Along the long axis $(z)$ we apply a constant temperature gradient and fixed antiparallel boundary conditions forcing a DW into the system. Analytically, we will treat the model in the one-dimensional limit. In our accompanying simulations the model has a size of $8 \mathrm{~nm} \times 8 \mathrm{~nm} \times 512 \mathrm{~nm}$ discretized with a cell size of $1 \mathrm{~nm}$. The DW is initially in the center of the wire with a temperature of $300 \mathrm{~K}$ (see Fig. 3). All numerical methods based on solving Eq. (1) for each cell are similar to Ref. [5].

As argued above, in a temperature gradient the DW moves towards increasing temperatures. Figure 3 shows that though the general shape of the wall remains constant, its amplitude decreases due to the fact that the equilibrium magnetization itself is temperature dependent. While for lower temperature gradients after an acceleration phase the DW is restricted to a constant plane (as shown in Fig. 3) for larger thermal gradients, the motion of the DW is accompanied by precession (not shown). This effect is the so-called Walker breakdown, well known from DW motion by applied fields or spin-polarized currents [17,24] and

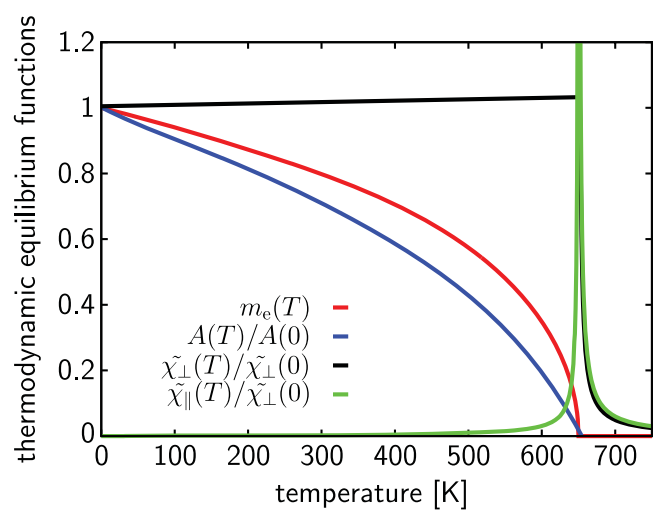

FIG. 2 (color online). Temperature dependence of the material parameters of the LLB equation. 

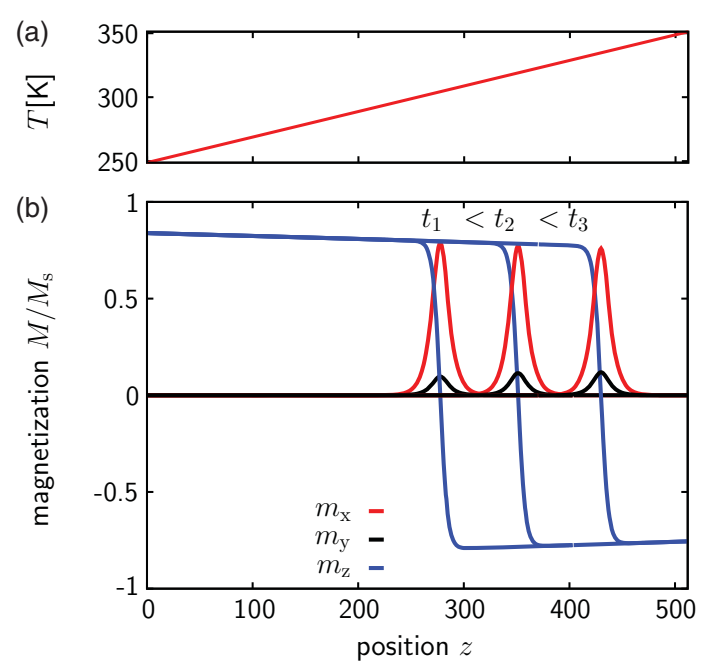

FIG. 3 (color online). DW motion in a thermal gradient. (a) Temperature gradient and (b) snapshots of the DW profile at different times.

already discussed in Ref. [5] for thermally driven DW motion. In the following we discuss an analytical solution of the DW velocity as well as the Walker threshold derived within the framework of the LLB equation. Details of the calculation are explained in the Supplemental Material [25]. Considering a 1D model with a thermal gradient as well as an external field in the $z$ direction, the calculations lead to the following equations of motion for the out of plane angle,

$$
\dot{\phi}=\gamma\left[H_{z}-\frac{2}{M_{s} m_{e} \delta} \frac{\partial A}{\partial z}-\frac{\alpha_{\perp}(1-k) \sin (2 \phi)}{2 \tilde{\chi}_{\perp}}\right],
$$

and for the velocity of the DW,

$$
v_{D}=\frac{\gamma}{\alpha_{\perp} M_{s}}\left(1+\frac{\alpha_{\perp}^{2}}{m_{e}^{2}}\right)\left(\delta M_{s} m_{e} H_{z}-2 \frac{\partial A}{\partial z}\right)-\frac{\delta m_{e}}{\alpha_{\perp}} \dot{\phi}
$$

Here we have assumed a constant transverse domain wall profile during the movement with the temperaturedependent domain wall width $\delta$ [see Eq. (7) in [25]] and neglected dynamic deviations of the local magnetization $m(z)$ from its equilibrium value $m_{e}(z)$. Concerning the external field contribution to the DW velocity below the Walker breakdown, we note that in the low-temperature limit $T \rightarrow 0$ our result agrees with Eq. (28) of the original evaluation by Landau and Lifshitz [26]. From our main results [Eq. (4)] we find that the temperature gradient creates an effective field $H_{\text {therm }}$ that acts on the DW, similar to the applied magnetic field. By comparing these two contributions we find a field equivalent given by

$$
H_{\text {therm }}=-\frac{2}{\delta m_{e} M_{s}} \frac{\partial A}{\partial T} \frac{\partial T}{\partial z}
$$

Another interesting analogy is to the case of DW motion by spin transfer torque, where two additional torque terms, an adiabatic and nonadiabatic one, are added to the equation of motion. Comparing the resulting velocity formulas [Eq. (16) in Ref. [17] to Eq. (4)] below the Walker breakdown one can define the effective spin current equivalent,

$$
\beta u_{z}=\frac{\beta \mu_{B} j_{e} P^{0}}{e M_{s}}=-\frac{2 \gamma}{M_{s} m_{e}}\left(1+\frac{\alpha_{\perp}^{2}}{m_{e}^{2}}\right) \frac{\partial A}{\partial T} \frac{\partial T}{\partial z} .
$$

Here $\beta, \mu_{B}, j_{e}, e$, and $P^{0}$ represent the nonadiabaticity factor, the Bohr magneton, the electrical current density, the charge of the electron, as well as the spin polarization of the electrical current.

From now on we focus solely on thermally driven DW motion without external fields. The corresponding Walker threshold $\tilde{A}_{w}=\partial A_{w} / \partial z$ for that case is calculated by assuming $\dot{\phi}=0$ and $\phi=\pi / 4$ in Eq. (3). The Walker threshold is then given by

$$
\tilde{A}_{w}=-\frac{m_{e} M_{s} \alpha_{\perp} \delta_{w}(1-k)}{4 \tilde{\chi}_{\perp}},
$$

with the corresponding DW width $\delta_{w}=\delta(\phi=\pi / 4)$.

For the average velocity of the DW, we obtain then

$$
\begin{aligned}
v_{D}= & -\frac{2 \gamma}{M_{s} \alpha_{\perp}} \frac{\partial A}{\partial z}\left(1+\frac{\alpha_{\perp}^{2}}{m_{e}^{2}}\right) \\
& -\frac{\gamma \delta_{w} m_{e}(1-k)}{2 \tilde{\chi}_{\perp}} \sqrt{\left(\frac{\partial A / \partial z}{\tilde{A}_{w}}\right)^{2}-1,}
\end{aligned}
$$

where the second square-root contribution representing $\left(\delta m_{e} / \alpha_{\perp}\right) \dot{\phi}$ occurs only above the Walker threshold.

In Fig. 4 the resulting DW velocities versus strength of the temperature gradient are plotted for different damping constants and anisotropy ratios $k$. Equation (8) is compared to our corresponding numerical simulations. In general we obtain a good agreement in all cases including the correct calculation of the Walker breakdown. The fact that the numerical results are slightly higher below and smaller above the Walker breakdown as compared to the analytical ones is due to an additional driving force caused by $m(z) \neq m_{e}(z)$, which has been neglected in the analytical model [25].

Because of the temperature dependence of $\tilde{\chi}_{\perp}, \alpha_{\perp}, m_{e}, \delta$ as well $\tilde{A}_{w}$ in Eq. (8) the velocity of the DW does not remain constant during the movement along the temperature gradient. For comparison to the numerical results in Fig. 4 , as input to these quantities, we therefore have used 


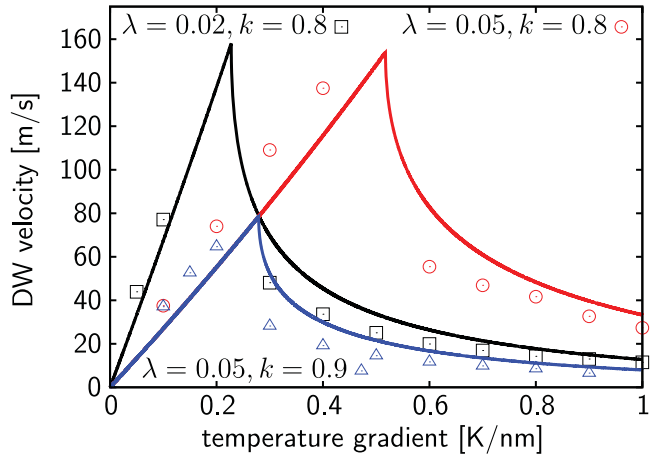

FIG. 4 (color online). Comparison between analytical (solid lines) and numerical results (data points) for the velocities of the DW for different damping constants and anisotropy ratios $k$.

the temperature at half of the covered distance. We illustrate the effect of the temperature variation in Fig. 5, where the temperature dependence of the DW velocity $(\lambda=0.02$, $k=0.8$ ) for different strengths of the thermal gradient is plotted together with the maximal possible velocity reached directly at the Walker breakdown

$$
v_{\max }=\frac{\gamma m_{e} \delta_{w}(1-k)}{2 \tilde{\chi}_{\perp}}\left(1+\frac{\alpha_{\perp}^{2}}{m_{e}^{2}}\right) .
$$

Mainly caused by the increase of $d A / d T$ with increasing temperature, the velocity of the DW is monotonically increasing below the Walker breakdown and decreasing above it. Since the Walker-breakdown itself is decreasing with temperature, dependent on the strength of the gradient, at a certain temperature the moving wall always starts to precess. For the realistic assumption of low damping, $\alpha^{2} \ll 1$, the maximum DW velocity [Eq. (9)] remains proportional to the difference between the hard and intermediate axis $(1-k)$, but becomes independent of the damping $\alpha$ as well as of the derivative of the exchange stiffness $\partial A / \partial T$ (see Fig. 4). Combining thermal gradients with external fields leads to higher velocities, but no change of the maximum DW velocity at the Walker breakdown. Note that we avoid the critical region in Fig. 5 since close to $T_{C}$ the wall becomes linear (see Fig. 1 and Refs. [11-13]) and the approximations made in the analytical model are no longer valid.

For a simpler analytical estimation of DW velocities that can be expected below the Walker breakdown, it is reasonable to linearize the temperature dependence of the exchange stiffness as $(d A / d T) \approx A(0) / T_{C}$ and approximate $\alpha^{2} \ll 1$ for low damping to obtain

$$
v_{D}=-\frac{2 \gamma}{M_{s}} \frac{1}{\alpha_{\perp}} \frac{A(0)}{T_{C}} \frac{\partial T}{\partial z} .
$$

In Refs. $[9,10]$ the DW velocity in this regime has been calculated from a microscopic point of view, assuming

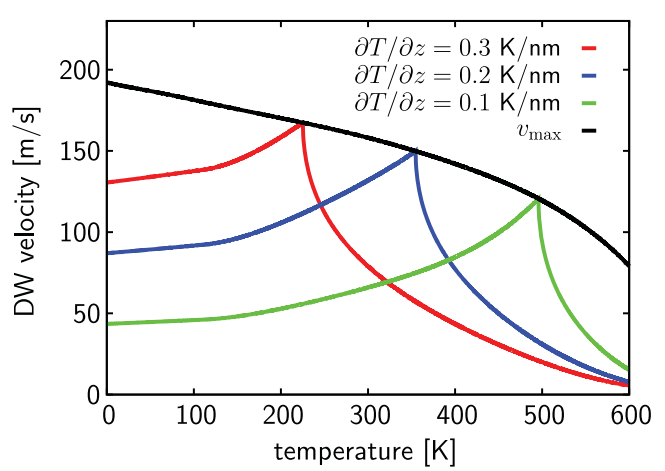

FIG. 5 (color online). Temperature dependence of the DW velocities for different strengths of the thermal gradient and of the maximum DW velocity at the Walker breakdown.

angular momentum transfer from the magnonic spin current only. Including the assumption $\alpha=\beta$ made by the authors, Eq. (1) in Ref. [9] can be written as

$$
\tilde{v}_{D}=\frac{\gamma k_{B}}{\alpha M_{s} 6 \pi^{2} \lambda_{m}} \frac{\partial T}{\partial z}
$$

Here, $\lambda_{m}$ represents the wavelength of the thermally excited magnons where the authors assume $\lambda \approx 6 \mathrm{~nm}$. Comparing Eq. (10) with Eq. (11) we note that both results agree in the proportionality of the DW velocity to $v_{D} \propto(\partial T / \partial z)$ $\left(\gamma / M_{s} \alpha\right)$. Concerning the prefactors in Eq. (10), we assume a cubic structure with $A(0)=J / 2 a$, where $a$ is the lattice constant amd $J$ the nearest-neighbor exchange constant and use the mean-field approximation for $T_{C}=2 J / k_{B}$. By doing so we obtain the relation $v_{D} / \tilde{v}_{D}=\left(3 \pi^{2} \lambda / 2 a\right) \gg 1$. Hence, the presented entropic torque drives the DW much more than the magnonic effect discussed in Refs. $[9,10]$. As an example, with the used YIG-material parameters $[9,10]$ and a gradient of $20 \mathrm{~K} / \mathrm{mm}$ we obtain a DW velocity of $24.6 \mathrm{~mm} / \mathrm{s}$ as compared to $1.3 \mathrm{~mm} / \mathrm{s}$. Based on a different approach the authors in Ref. [27] also discuss the effect of the maximization of entropy for DW motion in temperature gradients. In contrast to Eq. (10), in Ref. [27] there is no analytical estimation of the DW velocity without computational effort possible. Using the same values for YIG and $\partial T / \partial z$ as discussed above, the authors in Ref. [27] obtain a DW velocity of $7.0 \mathrm{~mm} / \mathrm{s}$.

To summarize, we presented a theory for DW motion in a temperature gradient that considers thermodynamic principles, mainly the maximization of entropy. Calculating the DW velocity analytically we show that the effect of the entropic torques should be larger than the angular momentum transfer from the magnon current. Our argument is rather general and not restricted to transverse domain walls in ferromagnets. We expect similar entropic torques also to act on other magnetic textures as, e.g., Skyrmions $[28,29]$ or vortex walls, and in other materials like antiferromagnets, where the magnonic spin currents are 
expected to vanish because of the two compensating sublattices [30].

We thank the DFG for financial support through the SFB 767 and SPP 1538.

[1] G. E. W. Bauer, E. Saitoh, and B. J. van Wees, Nat. Mater. 11, 391 (2012).

[2] K. Uchida, S. Takahashi, K. Harii, J. Ieda, W. Koshibae, K. Ando, S. Maekawa, and E. Saitoh, Nature (London) 455, 778 (2008).

[3] C. M. Jaworski, J. Yang, S. Mack, D. D. Awschalom, J. P. Heremans, and R.C. Myers, Nat. Mater. 9, 898 (2010).

[4] K. Uchida et al., Nature (London) 9, 894 (2010).

[5] D. Hinzke and U. Nowak, Phys. Rev. Lett. 107, 027205 (2011).

[6] U. Ritzmann, D. Hinzke, and U. Nowak, Phys. Rev. B 89, 024409 (2014).

[7] P. Yan, X. S. Wang, and X. R. Wang, Phys. Rev. Lett. 107, 177207 (2011).

[8] X. G. Wang, G. H. Guo, Y. Z. Nie, G. F. Zhang, and Z. X. Li, Phys. Rev. B 86, 054445 (2012).

[9] W. Jiang et al., Phys. Rev. Lett. 110, 177202 (2013).

[10] A. A. Kovalev and Y. Tserkovnyak, Europhys. Lett. 97, 67002 (2012).

[11] D. Hinzke, N. Kazantseva, U. Nowak, O. Mryasov, P. Asselin, and R. Chantrell, Phys. Rev. B 77, 094407 (2008).

[12] J. Kötzler, D. A. Garanin, M. Hartl, and L. Jahn, Phys. Rev. Lett. 71, 177 (1993).

[13] N. Kazantseva, R. Wieser, and U. Nowak, Phys. Rev. Lett. 94, 037206 (2005).
[14] N. Kazantseva, D. Hinzke, U. Nowak, R. Chantrell, U. Atxitia, and O. Chubykalo Fesenko, Phys. Rev. B 77, 184428 (2008).

[15] K. Vahaplar, A. Kalashnikova, A. Kimel, D. Hinzke, U. Nowak, R. Chantrell, A. Tsukamoto, A. Itoh, A. Kirilyuk, and Th. Rasing, Phys. Rev. Lett. 103, 117201 (2009).

[16] U. Atxitia, O. Chubykalo Fesenko, J. Walowski, A. Mann, and M. Münzenberg, Phys. Rev. B 81, 174401 (2010).

[17] C. Schieback, D. Hinzke, M. Kläui, U. Nowak, and P. Nielaba, Phys. Rev. B 80, 214403 (2009).

[18] P. M. Haney and M. D. Stiles, Phys. Rev. B 80, 094418 (2009).

[19] K. M. Lebecki and U. Nowak, J. Appl. Phys. 113, 023906 (2013).

[20] D. A. Garanin, Phys. Rev. B 55, 3050 (1997).

[21] S. Hashimoto, and Y. Ochiai, J. Magn. Magn. Mater. 88, 211 (1990).

[22] V. Kamberský, P. de Haan, J. Šimšová, S. Porthun, R. Gemperle, and J.C. Lodder, J. Magn. Magn. Mater. 157 158, 301 (1996).

[23] H. B. Callen and E. Callen, J. Phys. Chem. Solids 27, 1271 (1966).

[24] N. L. Schryer and L. R. Walker, J. Appl. Phys. 45, 5406 (1974).

[25] See Supplemental Material at http://link.aps.org/ supplemental/10.1103/PhysRevLett.113.097201 for details of the derivation.

[26] L. Landau and E. Lifshitz, Phys. Z. Sowjetunion 8, 101 (1935).

[27] X. S. Wang and X. R. Wang, Phys. Rev. B 90, 014414 (2014).

[28] L. Kong and J. Zang, Phys. Rev. Lett. 111, 067203 (2013).

[29] M. Mochizuki, X.Z. Yu, S. Seki, N. Kanazawa, W. Koshibae, J. Zang, M. Mostovoy, Y. Tokura, and N. Nagaosa, Nat. Mater. 13, 241 (2014).

[30] Y. Ohnuma, H. Adachi, E. Saitoh, and S. Maekawa, Phys. Rev. B 87, 014423 (2013). 